\title{
High urea and pregnancy or conception in dairy cows: A meta-analysis to define the appropriate urea threshold
}

\author{
D. Raboisson, ${ }^{1}$ A. Albaaj, G. Nonne, and G. Foucras \\ IHAP, Université de Toulouse, INRA, ENVT, Toulouse, France
}

\section{ABSTRACT}

Dietary proteins play an important role in reproduction, and increased dietary crude proteins, increased degradability of dietary proteins, and elevated blood or milk urea have been associated with decreased conception and pregnancy in many studies. The aim of this work was to provide a meta-analysis on the relationship between high milk or blood urea and pregnancy or conception, with a focus on defining the appropriate urea threshold associated with this issue. The meta-analysis included 61 different models from 21 papers. The thresholds of urea tested in the various models were built by steps of $1 \mathrm{~m} M$ urea. This constructed variable reduced heterogeneity by $61 \%$ in the meta-regression. The meta-analysis showed $43 \%$ lower odds of pregnancy or conception (odds ratio $=0.57 ; 95 \%$ confidence interval $=0.45-0.73)$ in cases where urea was $\geq 7.0 \mathrm{mM}$ in the blood (plasma urea nitrogen $=19.3 \mathrm{mg} / \mathrm{dL}$ ) or where urea was $\geq 420 \mathrm{mg} / \mathrm{L}$ in the milk compared with where urea values were lower. This threshold is the most suitable with regard to pregnancy or conception success, even if a threshold of $6.5 \mathrm{~m} M$ cannot be excluded with certainty. The results also highlighted the possibility of a stronger association between high urea concentrations and pregnancy or conception when high nitrogen exposure occurs before artificial insemination compared with after artificial insemination, but this possibility needs to be further studied. Whether the present results also apply to extensively pasture-based countries remains to be determined.

Key words: dairy cow, urea, nitrogen, reproduction

\section{INTRODUCTION}

Protein intake is an important determinant of dietary balance. Negative energy balance (NEB) is recognized as a significant challenge that dairy cows face during the early postpartum period. Diets that exceed the

Received September 17, 2016.

Accepted May 11, 2017.

${ }^{1}$ Corresponding author: d.raboisson@envt.fr

requirements for rumen degradation or contain nondegradable protein are often observed in the field (Butler, 1998). Urea is a good indicator of energy or protein imbalance and is a sensitive indicator of protein utilization efficiency (Kenny et al., 2002). Its small molecular size allows it to easily circulate in all fluids, and its concentration values are well correlated between milk and blood. Both milk and blood urea concentrations can be used to evaluate the nitrogen diet status of animals (Oltner and Wiktorsson, 1983; Baker et al., 1995). Dietary proteins play an important role in reproduction, and increased dietary CP, increased degradability of dietary proteins, and elevated blood or milk urea have been associated with decreased conception and pregnancy despite studies failing to highlight this association. Several previous studies (Westwood et al., 1998b; Leroy et al., 2008a,b) reviewed these relationships as well as the physiopathology behind the epidemiological link. In brief, diets high in dietary protein may interact with reproductive efficiency through (1) increased NEB linked to higher production and energy cost of desamination in the liver, (2) potential toxicity of the direct by-products of protein catabolism for the oocyte and the embryo, (3) prevention of the natural increase in uterine $\mathrm{pH}$ after ovulation and changes in the ionic composition of uterine fluid, (4) changes in $\mathrm{PGF}_{2 \alpha}$ secreted by the endometrial tissue, and (5) changes in the motility of spermatozoa (Butler, 1998; Westwood et al., 1998b; Melendez et al., 2003; Leroy et al., 2008a,b). The NEB promoted by high dietary protein also affects reproduction, including changes in the IGF-1, LH, and progesterone profiles (Leroy et al., 2008a). Interestingly, it seems that changes in dietary protein levels rather than high dietary protein levels alone may be involved in decreased reproductive performance. Lactating dairy cows can metabolically adapt to a prolonged high intake of quickly degradable protein, probably by opposing the adverse effects of longterm high concentrations of protein on embryo growth (Dawuda et al., 2002; Laven et al., 2004). There is still some uncertainty regarding whether the effect of high urea on reproduction occurs mostly during the period before, surrounding, or after AI (Hammon et al., 2000; 
Leroy et al., 2008b). The success of embryo transfer was decreased when the donors were fed high levels of dietary protein, whereas the result was not related to the urea nitrogen status of the recipients, suggesting a higher sensitivity of reproductive success to the factors preceding AI (Rhoads et al., 2006). The timing and duration of exposure need to be further studied, and their association with reproduction may depend on the parameter measured.

Many of the studies analyzing the association between dietary protein levels and reproduction used blood or milk urea (or urea nitrogen) as the gold standard to evaluate dietary nitrogen status. These studies were not specifically designed to address the association between urea concentration and pregnancy or conception, and the thresholds of urea used or defined in these studies were heterogeneous and arbitrary. Thus, the threshold of urea to be used in the field is ambiguous. Reviews on this topic have not focused on the threshold of urea or on the relative risk of decreased reproductive performance. This omission is concerning because these same studies are typically used to define the threshold to be achieved and to determine the relative risk of outcomes. This question has been intensively studied for subclinical ketosis (Raboisson et al., 2014). A quantitative review published in the 1990s included a small number of studies available on the topic. This work focused on conception (Westwood et al., 1998b). A review performed in 2003 gathered the available studies and highlighted the heterogeneity in the proposed thresholds of BUN or plasma urea nitrogen (PUN) for fertility but did not perform a formal meta-analysis (Melendez et al., 2003). Another study on reproduction performance did not evaluate urea but did evaluate dietary protein (Lean et al., 2012). The aim of this work was to provide a meta-analysis regarding the relationship between high milk or blood urea and reproductive performance, to propose the urea threshold for consideration in the field, and to clearly quantify the association between urea and pregnancy or conception.

\section{MATERIALS AND METHODS}

A literature search and screening process was conducted using the PubMed (http://www.ncbi.nlm.nih. gov/pubmed), CABI (www.cabi.org), and Google Scholar (http://scholar.google.com/) search engines to create a data set of papers using the key words "crude protein," "urea," "plasma urea nitrogen," "serum urea nitrogen," "reproduction," "conception," and "pregnancy" separately or in combination. New papers referenced by at least 1 of the papers identified in the previous step were also included.

\section{Inclusion and Exclusion Criteria}

To be included in the data set, the papers must have examined the odds ratios (OR) or equivalent of various reproductive performances, such as conception at service, pregnancy success, and so on, for cows or groups of cows with diets containing varying levels of urea or urea nitrogen. At this stage, no criteria regarding experimental design, including the number of animals per group, breed, urea measure (milk or blood), or use of hormones or synchronization protocols, were considered. Two types of studies were included: some studies reported the OR of reproduction performance change in the event of urea change (case 1), whereas others expressed the association differently but allowed us to obtain an expression for the OR through the construction of contingency tables (case 2). For this second situation, a temporary data set was implemented with the records of the mean urea values of groups of animals and the corresponding reproductive performances. Studies with in vitro experimental designs or with results without a clear estimation of the mean urea value for the different groups (with or without exposure to urea) were not included. Publications through June 2015 were included. All urea or nitrogen values were standardized (by dividing the urea nitrogen by 0.46 if BUN, PUN, or MUN) and are expressed in millimoles per liter. The models included at this stage refer to various outcomes: pregnancy after service $(\mathrm{n}=28)$, conception after service $(n=18)$, conception after the first service $(n=15)$, number of AI attempts before successful AI $(n=8)$, interval from calving to first AI $(\mathrm{n}=6)$, percentage of oocytes with division $(\mathrm{n}=12)$, percentage of embryos that reach the blastocyst stage $(\mathrm{n}=5)$, and viability of embryos $(\mathrm{n}=7)$. Because raw data must be distributed as a 5-class categorical variable for the meta-regression, only outcomes with a minimal number of raw data (i.e., only the first 3 outcomes) were retained for the subsequent part of the study (Supplemental Tables S1 and S2; https://doi.org/10.3168/jds.2016-12009). They were collected and labeled "pregnancy or conception." Descriptive data are given in Supplemental Table S3.

Twenty-one papers were selected. Most of the papers studied several outcomes or included more than 1 urea threshold, and 61 different models were recorded directly from the literature (case $1, \mathrm{n}=23$ ) or through the implementation of a contingency table (case $2, \mathrm{n}=$ 38). The final data set included the numbers of cows and herds studied, the energy density of the diet, the $\mathrm{CP}$ of the diet, the average milk production, the period (before, after, or both) of exposure to a high nitrogen level with respect to AI, the duration of high nitrogen level exposure, the date of sampling with respect to AI, 
the source used (blood or milk), whether pregnancy or conception was determined, the use of hormones or a synchronization protocol, the statistical method used (logistic regression, Poisson regression, raw data with contingency table), the threshold of urea used to classify the animals, the value of the OR or the change in the outcome and its $95 \%$ confidence interval (CI), the standard error or standard deviation, and the covariates included within the given models. The raw data of case 2 were modified for presentation as case 1 (OR calculated using temporary contingency tables). The threshold of urea to be considered was defined as the average of the mean urea values of the 2 groups of animals, and a variable gap was defined as the difference between the high or low urea mean values of the 2 groups of animals. The variable continuous threshold was then classified as the variables THRES and THRESbis according to the definitions reported in Table 1. The THRES and THRESbis are the categorical variables built with blood and milk urea concentration.

\section{Statistical Analysis: Meta-Analysis}

A meta-analysis was conducted on the extracted outcomes using the Metafor package (Viechtbauer, 2010) of R (version 3.0.2; R Foundation for Statistical Computing, Vienna, Austria). A fixed-effects model and a random-effects model were first used to estimate the log-effect size and its 95\% CI and statistical significance. This process was conducted separately for each class of THRES or THRESbis as a reference. The inconsistency of results among trials was quantified using both Cochran's Q test and the $I^{2}$ statistic (Higgins et al., 2003). A value greater than $50 \%$ indicated substantial heterogeneity. If evidence of heterogeneity was found, a meta-regression analysis was subsequently performed to explore the sources of heterogeneity using the log-individual effect size for each trial as the outcome and a fixed-effects model or mixed-effects model with the random moderator study. The meta-regression was conducted by screening for the moderators period of exposure, duration of high nitrogen level exposure, date of sampling, source (blood or milk), method used to determine pregnancy, use of hormones or synchronization protocol, and gap (Table 2 ). The $\tau^{2}$ values of the models, with or without moderators, were compared to explain the decrease in heterogeneity that occurred when the moderator was included in the model. Here, $\tau^{2}$ denoted the amount of residual heterogeneity among the true effects - that is, the variability among the true effects that was not accounted for by the moderators included in the model. Forest plots were used to display the estimated effect size with its $95 \%$ CI and the final
Table 1. Raw data of the categories of urea THRES and THRESbis ${ }^{1}$

\begin{tabular}{lccrc}
\hline & \multicolumn{2}{c}{ Urea } & & \\
\cline { 2 - 3 } Category & Blood $(\mathrm{m} M)$ & Milk $(\mathrm{mg} / \mathrm{L})$ & $\mathrm{n}$ & Mean odds ratio (SD) \\
\hline THRES & $\leq 4.9$ & $\leq 294$ & 16 & $1.25(0.98)$ \\
& $5-5.9$ & $300-354$ & 13 & $0.85(0.51)$ \\
& $6-6.9$ & $360-414$ & 15 & $0.85(0.34)$ \\
& $7-7.9$ & $420-474$ & 10 & $0.47(0.30)$ \\
THRESbis & $\geq 8$ & $\geq 480$ & 7 & $0.32(0.28)$ \\
& $\leq 4.4$ & $\leq 264$ & 15 & $1.28(1.00)$ \\
& $4.5-5.4$ & $270-324$ & 8 & $1.05(0.54)$ \\
& $5.5-6.4$ & $330-384$ & 10 & $0.71(0.31)$ \\
& $6.5-7.4$ & $390-444$ & 18 & $0.69(0.39)$ \\
& $\geq 7.5$ & $\geq 450$ & 10 & $0.35(0.25)$ \\
\hline
\end{tabular}

${ }^{1}$ THRES and THRESbis are the categorical variables built with blood and milk urea concentrations, according to thresholds defined in the table.

meta-regression adjustments (in gray). A sensitivity analysis was performed using funnel plots and influential case diagnostics. It included the analyses of externally standardized residuals, DFFITS (difference in fit) values, Cook's distances, covariance ratios, estimates of $\tau^{2}$ and test statistics for (residual) heterogeneity when each study was removed in turn, hat values, and weights for the studies examining the odds of each outcome in the case of high urea.

\section{RESULTS}

The descriptive statistics showed OR above 1 for low-urea classes of THRES and THRESbis and mean OR below 1 for high-urea classes (Table 1). Descriptive statistics for other variables are reported in Table 2 . The heterogeneity of the data set describing the association between pregnancy or conception and high urea (Supplemental Table S1; https://doi.org/10.3168/ jds.2016-12009) was high $\left(I^{2}=82 \% ; 95 \%\right.$ CI $=53-93$; Q statistics: $\chi^{2}=111$, degrees of freedom $=60, P<$ 0.001). The moderators THRES or THRESbis reduced

Table 2. Raw data of other variables

\begin{tabular}{llrl}
\hline Item & Category & n & $\begin{array}{l}\text { Mean odds } \\
\text { ratio (SD) }\end{array}$ \\
\hline Period of exposure & Before and after AI & 34 & $0.65(0.44)$ \\
& Before AI & 7 & $0.70(0.46)$ \\
Date of sampling & After AI & 20 & $1.19(0.89)$ \\
& Before and after AI & 23 & $0.74(0.56)$ \\
Before AI & 13 & $0.59(0.40)$ \\
Source & After AI & 25 & $1.04(0.80)$ \\
Synchronization & Blood & 38 & $0.80(0.69)$ \\
Pregnancy or conception & Milk & 23 & $0.88(0.62)$ \\
& AI of cows in heat & 53 & $0.84(0.69)$ \\
& Synchronization & 8 & $0.79(0.48)$ \\
& Pregnancy & 28 & $0.62(0.49)$ \\
& Conception & 18 & $1.16(0.80)$ \\
& Conception at first AI & 15 & $0.83(0.64)$ \\
\hline
\end{tabular}


the heterogeneity by 61 and $41 \%$, respectively (Table 3 , models 1 and 4). Using blood urea of 6 to $6.99 \mathrm{mM}$ as a reference showed $43 \%$ lower odds of pregnancy or conception $(\mathrm{OR}=0.57 ; 95 \% \mathrm{CI}=0.45-0.73)$ when the urea was above $7.0 \mathrm{~m} M$. The decrease in odds of pregnancy or conception was even greater if the urea was above $8.0 \mathrm{mM}$ (mixed-effects model 1). Using urea of 6.5 to $7.49 \mathrm{~m} M$ as a reference showed $33 \%$ lower odds of pregnancy or conception when the urea was above 7.5 $\mathrm{m} M$ (mixed-effects model 4) and a significant increase in odds of pregnancy or conception for urea of 5.5 to $6.49 \mathrm{~m} M$ (fixed-effects model 4). Using urea $\leq 4.49$ or 5 to $5.99 \mathrm{~m} M$ instead of 6 to $6.99 \mathrm{~m} M$ as a reference showed a significant decrease in the odds of pregnancy or conception for urea $\geq 7 \mathrm{~m} M$ (Supplemental Table S4; https://doi.org/10.3168/jds.2016-12009). Using urea of 7 to $7.99 \mathrm{mM}$ as a reference showed a significant decrease in the odds of pregnancy or conception for urea $\geq 8 \mathrm{~m} M$ for the fixed-effects model. Similar results were observed for THRESbis (Supplemental Table S5; https://doi.org/10.3168/jds.2016-12009).

Including any other moderator in models 1 or 4 did not further decrease the heterogeneity of the data set and did not change the results (coefficients and $P$-values) observed for the moderator THRES or THRESbis. The moderators synchronization and source (milk or blood) were not significant for either the fixed-effects model or the mixed-effects model. In the fixed-effects model, exposure to high urea after AI was associated with improved odds of pregnancy or conception compared with exposure before and after AI (Table 3, model 2), and the OR of pregnancy or conception in cases of sampling before AI was $0.92(95 \%$ CI $=0.86-0.99)$ compared with surrounding AI (Table 3, model 3). The inclusion of the moderator pregnancy or conception in models 1 or 4 showed an OR for success of AI of 0.81 (95\% CI $=0.73-0.90)$ compared with conception at AI for the fixed-effects model. This finding led us to run models 1 and 4 on the 3 subpopulations of the moderator pregnancy or conception (Table 2). These 3 submodels clearly showed coefficients of interest similar to models 1 to 4 except for the nonsignificant association between THRES or THRESbis and the class conception. Models 1 to 4 performed using subpopulations of raw data cases (i.e., case 1 or 2 separately) showed results similar to those reported in Table 3.

Table 3. Results of meta-regressions

\begin{tabular}{|c|c|c|c|c|}
\hline \multirow[b]{2}{*}{ Model } & \multirow[b]{2}{*}{ Variable $^{1}$} & \multirow[b]{2}{*}{ Category } & \multicolumn{2}{|c|}{ Odds ratio of pregnancy or conception (95\% CI) } \\
\hline & & & Fixed-effects model & Mixed-effects model \\
\hline \multirow{4}{*}{1} & THRES & $\leq 4.9 \mathrm{mM}(\leq 294 \mathrm{mg} / \mathrm{L})$ & $0.93(0.87-1.00)$ & $0.95(0.89-1.02)$ \\
\hline & & $\overline{5}-5.9 \mathrm{~m} M(\overline{3} 00-354 \mathrm{mg} / \mathrm{L})$ & $0.95(0.89-1.01)$ & $0.96(0.90-1.02)$ \\
\hline & & 6-6.9 mM (360-414 mg/L) & Referent & Referent \\
\hline & & $7-7.9 \mathrm{~m} M(420-474 \mathrm{mg} / \mathrm{L})$ & $0.47(0.39-0.56)$ & $0.57(0.45-0.73)$ \\
\hline \multirow{7}{*}{2} & THRES & $\leq 4.9 \mathrm{~m} M(\leq 294 \mathrm{mg} / \mathrm{L})$ & $0.94(0.89-1.00)$ & $0.95(0.89-1.02)$ \\
\hline & & $5-5.9 \mathrm{~m} M(300-354 \mathrm{mg} / \mathrm{L})$ & $0.96(0.90-1.01)$ & $0.96(0.91-1.03)$ \\
\hline & & $6-6.9 \mathrm{~m} M(360-414 \mathrm{mg} / \mathrm{L})$ & Referent & Referent \\
\hline & & $7-7.9 \mathrm{mM}(420-474 \mathrm{mg} / \mathrm{L})$ & $0.50(0.41-0.61)$ & $0.59(0.46-0.74)$ \\
\hline & & $\geq 8 \mathrm{~m} M(\geq 480 \mathrm{mg} / \mathrm{L})$ & $0.28(0.20-0.40)$ & $0.49(0.29-0.82)$ \\
\hline & Period of exposure & Before and after AI & Referent & Referent \\
\hline & & Before AI & $0.96(0.77-1.21)$ & $0.97(0.56-1.65)$ \\
\hline \multirow{5}{*}{3} & & $7-7.9 \mathrm{~m} M(420-474 \mathrm{mg} / \mathrm{L})$ & $0.48(0.40-0.58)$ & $0.58(0.46-0.74)$ \\
\hline & & $\geq 8 \mathrm{~m} M(\geq 480 \mathrm{mg} / \mathrm{L})$ & $0.27(0.19-0.38)$ & $0.49(0.29-0.82)$ \\
\hline & Date of sampling & $\bar{B}$ efore and after AI & Referent & Referent \\
\hline & & Before AI & $0.92(0.86-0.99)$ & $0.89(0.55-1.44)$ \\
\hline & & After AI & $1.10(0.95-1.27)$ & $1.21(0.77-1.91)$ \\
\hline \multirow[t]{6}{*}{4} & Intercept & & $0.81(0.73-0.91)$ & $0.70(0.55-0.89)$ \\
\hline & THRESbis & $\leq 4.4 \mathrm{~m} M(\leq 264 \mathrm{mg} / \mathrm{L})$ & $1.11(0.99-1.25)$ & $1.05(0.89-1.24)$ \\
\hline & & $4.5-5.4 \mathrm{mM}(270-324 \mathrm{mg} / \mathrm{L})$ & $1.12(1.00-1.26)$ & $1.05(0.89-1.23)$ \\
\hline & & $5.5-6.4 \mathrm{~m} M(330-384 \mathrm{mg} / \mathrm{L})$ & $1.15(1.02-1.29)$ & $1.07(0.91-1.26)$ \\
\hline & & $6.5-7.4 \mathrm{~m} M(390-444 \mathrm{mg} / \mathrm{L})$ & Referent & Referent \\
\hline & & $\geq 7.5 \mathrm{~m} M(\geq 450 \mathrm{mg} / \mathrm{L})$ & $0.39(0.29-0.52)$ & $0.66(0.44-0.99)$ \\
\hline
\end{tabular}

${ }^{1}$ THRES and THRESbis are the categorical variables built with blood and milk urea concentrations, according to thresholds defined in the table. 

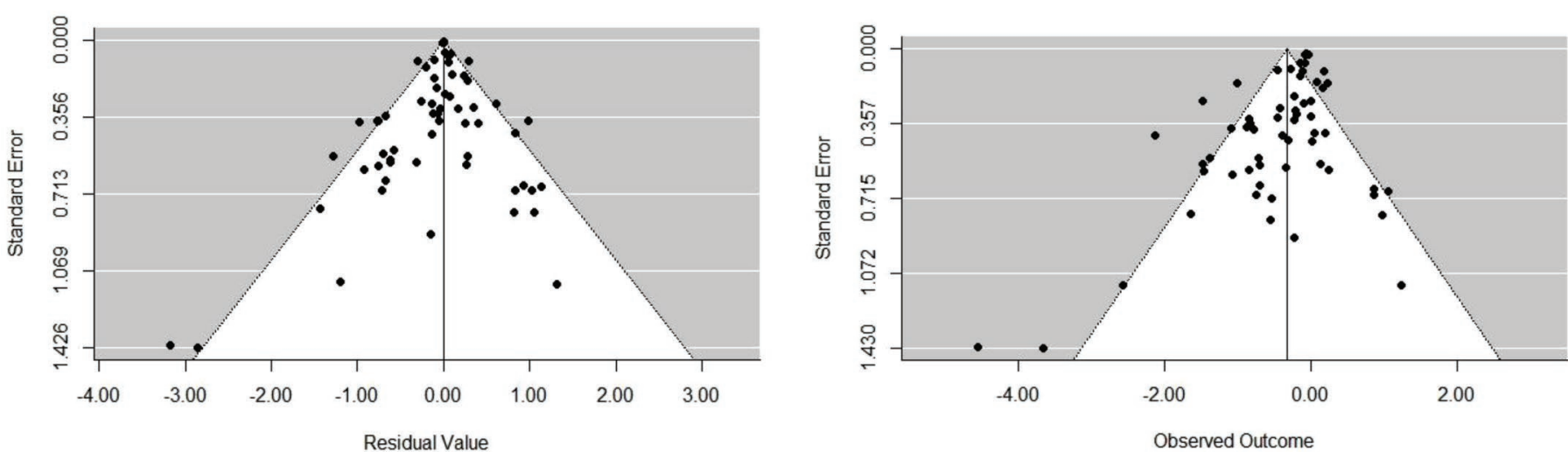

Figure 1. Funnel plots for the meta-regressions. Funnel plots of residual and observed risk of conception or pregnancy (displayed on the horizontal axis) for cows in cases of urea $\geq 7.0 \mathrm{~m} M$ in blood, including line of no effect and pseudo $95 \%$ confidence interval.

The sensitivity analysis showed no evidence of publication bias in the funnel plot (Figure 1) and no outlier for the meta-regressions, but doubt exists for 3 to 5 raw data points (Supplemental Figure S1; https://doi. org/10.3168/jds.2016-12009). Excluding these data did not lead to significant changes in either the significance of the results or the coefficients.

\section{DISCUSSION}

This work clearly demonstrates $43 \%$ lower odds of pregnancy or conception $(\mathrm{OR}=0.57 ; 95 \% \mathrm{CI}=0.45-$ $0.73)$ in cases of urea $\geq 7.0 \mathrm{~m} M$ in blood (PUN $=19.3$ $\mathrm{mg} / \mathrm{dL}$ ) or urea $\geq 420 \mathrm{mg} / \mathrm{L}$ in milk compared with lower values. The OR can reach 0.55 in cases of very high urea $(\geq 7.0 \mathrm{~m} M$ or $420 \mathrm{mg} / \mathrm{L} ; \mathrm{BUN}=19.3 \mathrm{mg} / \mathrm{dL})$ compared with moderate cases (7-8 $\mathrm{m} M$ or $420-470$ $\mathrm{mg} / \mathrm{L} ; \quad \mathrm{BUN}=19.3-22.1 \mathrm{mg} / \mathrm{dL})$. The association between high urea and decreased pregnancy or conception appeared to be even stronger when urea above the threshold of $7 \mathrm{~m} M$ was considered high. The nonsignificant differences between classes of urea of 7 to 8 and $>8 \mathrm{~m} M$ (Supplemental Table S4, model D; https://doi. org/10.3168/jds.2016-12009) are likely to be linked to the low statistical power because of the small number of cases in the highest urea class $(\mathrm{n}=7)$. The tendency toward better pregnancy or conception in the reference class (urea $=6-7 \mathrm{~m} M$ or $360-420 \mathrm{mg} / \mathrm{L}$ ) compared with lower classes, even if not significant (Tables 2 and 3 ), suggests a favorable moderate urea concentration and shows that no evidence supports pursuing a very low urea value to improve pregnancy or conception. This tendency was not observed for THRESbis (model 4 ), and only a few very low values of urea were included in the meta-analysis. The threshold $7.0 \mathrm{mM}$ (model 1) is preferred to the threshold $6.5 \mathrm{~m} M$ (model 4) because the heterogeneity was lower with the inclusion of THRES compared with THRESbis. Lower odds of pregnancy or conception have already been observed in cases of urea $\geq 6.5 \mathrm{mM}$ compared with lower values (model 4). The thresholds proposed here are (1) in accordance with those previously proposed in a review (6.9-7.2 $\mathrm{m} M$ of urea; BUN or MUN $=19-20 \mathrm{mg} / \mathrm{dL}$; Butler, 1998) but (2) above the milk urea concentrations that are usually recommended in France (250-350 $\mathrm{mg} / \mathrm{L}$ ) or the objective (milk urea $=250-305 \mathrm{mg} / \mathrm{L}$ or $\mathrm{MUN}=11.5-14.0 \mathrm{mg} / \mathrm{dL}$ ) proposed in a recent review (Melendez et al., 2003). Such differences may arise from whether the recommended urea values were defined through (1) optimal and efficient use of dietary nitrogen in the rumen, with adjustment in dietary proteins or rumen fermentable energy needed in case of greater values (Melendez et al., 2003), or (2) significant odds of deterioration in reproduction performance observed in cases of urea above a certain threshold, as proposed here and elsewhere (Butler, 1998).

The second main conclusion is related to the association between pregnancy or conception and the period of high nitrogen exposure (fixed-effects model 2) or date of sampling (fixed-effects model 3). The study suggests a stronger association between high urea and pregnancy or conception when the exposure occurs before AI compared with after AI. This result is in accordance with the literature (Hammon et al., 2000; Rhoads et al., 2006) despite the inconsistent results. The mixed-effects models were not significant: the results of the 2 previous fixed-effects models (2 and 3) ultimately rely on the structure of the data set alone, suggesting extreme caution in the interpretation of this second conclusion. The data available for this study did not allow a rigorous evaluation of the association between the timing of protein interventions and the establishment of conception or maintenance of pregnancy. Dairy cows were recognized to be able to adapt to high urea concentration without any deterioration in reproduction performance 
provided that the urea concentration remains constant at high values and that there is a wash-out period before AI (Westwood et al., 1998b). This situation may be why high urea is recognized as a risk factor (with thresholds and odds ratios as indicated in this study) in most Northern countries, where livestock systems are most sensitive to urea change due to characteristics of feeding systems. In contrast, Australasian-type pasture-based systems with lower yielding cows may be less affected or unaffected by this issue (Westwood et al., 1998a,b; Laven et al., 2007; Ordonez et al., 2007).

This study was performed as recommended (Viechtbauer, 2010; Kovalchik, 2013). The final meta-regression (and relative adjusted OR) retained was judged on the reduction of the heterogeneity relative to regression without the moderator. The reduced heterogeneity allowed by the moderator THRES or THRESbis was expected because most of the raw data were obtained with various mean values or thresholds of urea. Models 1 and 4 should be considered the reference models of this study. As recommended by Kovalchik (2013), both fixed-effects and mixed-effects models were reported in this work. The fixed-effects meta-regression is a description of the $k$ studies, and the random or mixed-effects meta-regression treats the $k$ studies as a sample of a larger universe of studies. Because raw data may arise from the same study, the mixed-effects model is more robust in this situation than the fixed-effects model. The sensitivity analysis and the investigation of outliers showed the robustness of the results.

The assumptions in the way the data set was implemented have been validated through the results of the models. First, the models included in the data set referred to 3 closely related outcomes (OR of pregnancy, conception, or conception at first AI). Neither the behavior of this moderator (pregnancy, conception, or conception at first AI) in the main model (significant only in the fixed-effects model) nor the behavior of the 3 submodels performed separately for pregnancy, conception, or conception at first AI (with results similar to models 1 to 4 ) suggested considering these 3 topics separately. Second, neither the submodel performed with the data from case 1 alone nor the inclusion of the moderator gap in models 1 or 4 suggested any bias linked to the building of the data set with the 2 main types of data. Merging the 2 data sets made it possible to obtain enough data in each of the classes for moderator THRES or THRESbis. Third, the nonsignificant moderator source is consistent with the good correlation observed between milk and blood urea and validated the experimental design of this work, which did not distinguish the milk or blood urea. This result is consistent with the literature (Butler et al., 1996).
Last, the CP and ME of the diets were not always reported in the studies, preventing their inclusion in the meta-regressions. This information might have helped highlight the complex relationship between energy and nitrogen supply.

\section{CONCLUSIONS}

This meta-analysis showed $43 \%$ lower odds of conception or pregnancy $(\mathrm{OR}=0.57 ; 95 \% \mathrm{CI}=0.45-0.73)$ in cases where urea was $\geq 7.0 \mathrm{~m} M$ in blood (PUN = $19.3 \mathrm{mg} / \mathrm{dL}$ ) or urea was $\geq 420 \mathrm{mg} / \mathrm{L}$ in milk compared with where urea values were lower. The results showed that this threshold is the most suitable with regard to pregnancy or conception, even if a threshold of 6.5 $\mathrm{m} M$ cannot be excluded with certainty. The results also highlighted the possibility of a stronger association between high urea and pregnancy or conception when the exposure occurs before AI compared with after AI, but this association needs to be confirmed through further studies. The results of this study reflect the data used in the study, which were obtained mostly from intensive production systems in the Northern Hemisphere and may not be applicable to Australasian-type pasturebased systems.

\section{REFERENCES}

Baker, L. D., J. D. Ferguson, and W. Chalupa. 1995. Responses in urea and true protein of milk to different protein feeding schemes for dairy cows. J. Dairy Sci. 78:2424-2434.

Butler, W. R. 1998. Review: Effect of protein nutrition on ovarian and uterine physiology in dairy cattle. J. Dairy Sci. 81:2533-2539.

Butler, W. R., J. J. Calaman, and S. W. Beam. 1996. Plasma and milk urea nitrogen in relation to pregnancy rate in lactating dairy cattle. J. Anim. Sci. 74:858-865.

Dawuda, P. M., R. J. Scaramuzzi, H. J. Leese, C. J. Hall, A. R. Peters, S. B. Drew, and D. C. Wathes. 2002. Effect of timing of urea feeding on the yield and quality of embryos in lactating dairy cows. Theriogenology 58:1443-1455.

Hammon, D. S., S. Wang, and G. R. Holyoak. 2000. Effects of ammonia during different stages of culture on development of in vitro produced bovine embryos. Anim. Reprod. Sci. 59:23-30.

Higgins, J. P., S. G. Thompson, J. J. Deeks, and D. G. Altman. 2003. Measuring inconsistency in meta-analyses. BMJ 327:557-560.

Kenny, D. A., P. G. Humpherson, H. J. Leese, D. G. Morris, A. D. Tomos, M. G. Diskin, and J. M. Sreenan. 2002. Effect of elevated systemic concentrations of ammonia and urea on the metabolite and ionic composition of oviductal fluid in cattle. Biol. Reprod. $66: 1797-1804$.

Kovalchik, S. 2013. Tutorial on meta-analysis in R. R useR! Conference 2013:204 p. http://www.edii.uclm.es/ useR-2013/Tutorials/ kovalchik/kovalchik_meta_tutorial.pdf.

Laven, R. A., P. M. Dawuda, R. J. Scaramuzzi, D. C. Wathes, H. J. Biggadike, and A. R. Peters. 2004. The effect of feeding diets high in quickly degradable nitrogen on follicular development and embryo growth in lactating Holstein dairy cows. Anim. Reprod. Sci. 84:41-52.

Laven, R. A., R. J. Scaramuzzi, D. C. Wathes, A. R. Peters, and T. J. Parkinson. 2007. Recent research on the effects of excess dietary nitrogen on the fertility of dairy cows. Vet. Rec. 160:359-362. 
Lean, I. J., P. Celi, H. Raadsma, J. McNamara, and A. R. Rabiee. 2012. Effects of dietary crude protein on fertility: Meta-analysis and meta-regression. Anim. Feed Sci. Technol. 171:31-42.

Leroy, J. L., G. Opsomer, A. Van Soom, I. G. Goovaerts, and P. E. Bols. 2008a. Reduced fertility in high-yielding dairy cows: Are the oocyte and embryo in danger? Part I. The importance of negative energy balance and altered corpus luteum function to the reduction of oocyte and embryo quality in high-yielding dairy cows. Reprod. Domest. Anim. 43:612-622.

Leroy, J. L., A. Van Soom, G. Opsomer, I. G. Goovaerts, and P. E. Bols. 2008b. Reduced fertility in high-yielding dairy cows: Are the oocyte and embryo in danger? Part II. Mechanisms linking nutrition and reduced oocyte and embryo quality in high-yielding dairy cows. Reprod. Domest. Anim. 43:623-632.

Melendez, P., A. Donovan, J. Hernandez, J. Bartolome, C. A. Risco, C. Staples, and W. W. Thatcher. 2003. Milk, plasma, and blood urea nitrogen concentrations, dietary protein, and fertility in dairy cattle. J. Am. Vet. Med. Assoc. 223:628-634.

Oltner, R., and H. Wiktorsson. 1983. Urea concentrations in milk and blood as influenced by feeding varying amounts of protein and energy to dairy cows. Livest. Prod. Sci. 10:457-467.

Ordonez, A., T. J. Parkinson, C. Matthew, C. W. Holmes, R. D. Miller, N. Lopez-Villalobos, J. Burke, and I. Brookes. 2007. Effects of application in spring of urea fertiliser on aspects of reproductive performance of pasture-fed dairy cows. N. Z. Vet. J. 55:69-76.

Raboisson, D., M. Mounie, and E. Maigne. 2014. Diseases, reproductive performance, and changes in milk production associated with subclinical ketosis in dairy cows: A meta-analysis and review. J. Dairy Sci. 97:7547-7563.

Rhoads, M. L., R. P. Rhoads, R. O. Gilbert, R. Toole, and W. R. Butler. 2006. Detrimental effects of high plasma urea nitrogen levels on viability of embryos from lactating dairy cows. Anim. Reprod. Sci. 91:1-10.

Viechtbauer, W. 2010. Conducting meta-analyses in R with the metafor package. J. Stat. Softw. 36:1-48.

Westwood, C. T., I. J. Lean, and R. C. Kellaway. 1998a. Indications and implications for testing of milk urea in dairy cattle: A quantitative review. Part 1. Dietary protein sources and metabolism. N Z. Vet. J. 46:87-96.

Westwood, C. T., I. J. Lean, and R. C. Kellaway. 1998b. Indications and implications for testing of milk urea in dairy cattle: A quantitative review. Part 2. Effect of dietary protein on reproductive performance. N. Z. Vet. J. 46:123-130. 